Corrected: Publisher correction

ARTICLE

DOI: $10.1038 /$ s41467-018-06649-5

\title{
Novel pleiotropic risk loci for melanoma and nevus density implicate multiple biological pathways
}

David L. Duffy (10 1, Gu Zhu', Xin Li ${ }^{2}$, Marianna Sanna ${ }^{3}$, Mark M. Iles ${ }^{4}$, Leonie C. Jacobs ${ }^{5}$, David M. Evans ${ }^{6,7}$, Seyhan Yazar (10 ${ }^{8}$, Jonathan Beesley ${ }^{1}$, Matthew H. Law (1) ${ }^{1}$, Peter Kraft ${ }^{9}$, Alessia Visconti (i) ${ }^{3}$, John C. Taylor (D) ${ }^{4}$, Fan Liu ${ }^{10}$, Margaret J. Wright (10 1, Anjali K. Henders ${ }^{1,17}$, Lisa Bowdler ${ }^{1}$, Dan Glass ${ }^{3}$, M. Arfan Ikram (D) ${ }^{11}$, André G. Uitterlinden 11,12, Pamela A. Madden ${ }^{13}$, Andrew C. Heath ${ }^{13}$, Elliot C. Nelson ${ }^{13}$, Adele C. Green (1) 1,14, Stephen Chanock (1) ${ }^{15}$, Jennifer H. Barrett (10 4, Matthew A. Brown (1) 7, Nicholas K. Hayward', Stuart MacGregor (10 1, Richard A. Sturm ${ }^{16}$, Alex W. Hewitt (10) ${ }^{8}$, Melanoma GWAS Consortium, Manfred Kayser (10 ${ }^{10}$, David J. Hunter ${ }^{9}$, Julia A. Newton Bishop ${ }^{4}$, Timothy D. Spector ${ }^{3}$, Grant W. Montgomery (1) 1,17, David A. Mackey (10 ${ }^{8}$, George Davey Smith ${ }^{6}$, Tamar E. Nijsten ${ }^{5}$, D. Timothy Bishop (1) ${ }^{4}$, Veronique Bataille ${ }^{3}$, Mario Falchi ${ }^{3}$, Jiali $\operatorname{Han}^{2}$ \& Nicholas G. Martin ${ }^{1}$

The total number of acquired melanocytic nevi on the skin is strongly correlated with melanoma risk. Here we report a meta-analysis of 11 nevus GWAS from Australia, Netherlands, UK, and USA comprising 52,506 individuals. We confirm known loci including MTAP, PLA2G6, and IRF4, and detect novel SNPs in KITLG and a region of 9q32. In a bivariate analysis combining the nevus results with a recent melanoma GWAS meta-analysis $(12,874$ cases, 23,203 controls), SNPs near GPRC5A, CYP1B1, PPARGC1B, HDAC4, FAM208B, DOCK8, and SYNE2 reached global significance, and other loci, including MIR146A and OBFC1, reached a suggestive level. Overall, we conclude that most nevus genes affect melanoma risk (KITLG an exception), while many melanoma risk loci do not alter nevus count. For example, variants in TERC and OBFC1 affect both traits, but other telomere length maintenance genes seem to affect melanoma risk only. Our findings implicate multiple pathways in nevogenesis.

\footnotetext{
${ }^{1}$ QIMR Berghofer Medical Research Institute, Brisbane, Australia. ${ }^{2}$ Department of Epidemiology, Richard M. Fairbanks School of Public Health, Melvin and Bren Simon Cancer Center, Indiana University, Indianapolis, IN 63110, USA. ${ }^{3}$ Department of Twin Research \& Genetic Epidemiology, St Thomas Hospital Campus, Kings College, London, UK. ${ }^{4}$ Section of Epidemiology and Biostatistics, Leeds Institute of Cancer and Pathology, University of Leeds, Leeds, UK. ${ }^{5}$ Department of Dermatology, Erasmus MC University Medical Centre Rotterdam, Rotterdam, The Netherlands. ${ }^{6}$ MRC Integrative Epidemiology Unit, University of Bristol, Bristol, UK. ${ }^{7}$ University of Queensland Diamantina Institute, Translational Research Institute, Brisbane, Australia. ${ }^{8}$ Centre for Ophthalmology and Vision Science, University of Western Australia and the Lions Eye Institute, Perth, Australia. ${ }^{9}$ Department of Epidemiology, Harvard T.H. Chan School of Public Health, Boston 02115 MA, USA. ${ }^{10}$ Department of Genetic Identification, Erasmus MC University Medical Centre Rotterdam, Rotterdam, The Netherlands. ${ }^{11}$ Department of Epidemiology, Erasmus MC, Rotterdam, Netherlands. ${ }^{12}$ Department of Internal Medicine, Erasmus MC, Rotterdam, Netherlands. ${ }^{13}$ Department of Psychiatry, Washington University School of Medicine, St. Louis, MO 63110, USA. ${ }^{14}$ Molecular Oncology Group, CRUK Manchester Institute, University of Manchester, Manchester, UK. ${ }^{15}$ Division of Cancer Epidemiology and Genetics, National Cancer Institute, Bethesda, MD, USA. ${ }^{16}$ Dermatology Research Centre, University of Queensland Diamantina Institute, Translational Research Institute, Brisbane, Australia. ${ }^{17}$ Present address: Institute for Molecular Bioscience, The University of Queensland, Brisbane, Australia. These authors contributed equally: Jiali Han and Nicholas G. Martin. Full list of members of the Melanoma GWAS Consortium is given at the end of this paper. Correspondence and requests for materials should be addressed to D.L.D. (email: David.Duffy@gimrberghofer.edu.au)
} 
T he incidence of cutaneous malignant melanoma (CM) has increased in populations of European descent in North America, Europe, and Australia due to long-term changes in sun exposure behavior, as well as screening ${ }^{1}$. The strongest CM epidemiological risk factor acting within populations of European descent is the number of cutaneous acquired melanocytic nevi, with risk increasing by $2-4 \%$ per additional nevus counted ${ }^{2}$. Nevi are benign melanocytic tumors usually characterized by a signature somatic $B R A F$ mutation. Their association with CM can be direct, in that a proportion of melanomas arise within a preexisting nevus (due to a "second hit" mutation), or indirect, where genetic or environmental risk factors for both traits are shared. Total nevus count is highly heritable $(60 \%-90 \% \text { in twins })^{3,4}$, but only a small proportion of this genetic variance is explained by loci identified so far $^{5-9}$. The known nevus count loci all have pleiotropic effects on $\mathrm{CM}$ risk $^{5-9}$, which implies both that nevus count loci are medically important and that a genetic analysis combining nevi and CM phenotypes will have increased statistical power. Here we present a new large nevus genome-wide association meta-analysis, and combine these results with those of a previously published meta-analysis of melanoma ${ }^{10}$.

\section{Results}

Nevus GWAS meta-analysis. Genome-wide single-nucleotide polymorphism (SNP) genotype data were available for a total of 52,806 individuals from 11 studies in Australia, UK, USA, and the Netherlands (Table 1), where nevus number had been measured by counting or ratings, by self or observer, and of the whole body or selected regions. Analyses show that these are measuring the same entity and are therefore combinable for GWAS (genomewide association study; see Supplementary Results). The genomic inflation factors were $\lambda=1.41$ and $\lambda_{1000}=1.008$ (Q-Q plot, Supplementary Fig. 1), consistent with polygenic inheritance and the total sample size. ${ }^{11}$ Five genomic regions contained association peaks that reached genome-wide significance in the nevus count meta-analysis (Fig. 1, Table 2, Supplementary Fig. 2),
MTAP/CDKN2A on chromosomes 9p21.3 (peak SNP, $P=2 \times 10$ ${ }^{-37}$ ) and $9 \mathrm{q} 31.1-2\left(P=1 \times 10^{-8}\right)$, IRF4 on chromosome $6 \mathrm{p}$ (peak SNP, $P=4 \times 10^{-37}$ ), in KITLG in the region of the known testicular germ cell cancer risk locus $\left(P=8 \times 10^{-9}\right)$, rs600951 over DOCK8 on chromosome 9p24.3 $\left(P=2 \times 10^{-8}\right)$, and PLA2G6 on chromosome $22\left(P=3 \times 10^{-18}\right)$. We have previously detected three of these in analyses using subsets of the meta-analysis sample $^{5,10}$. A SNP, rs251464, in PPARGC1B $\left(P=5 \times 10^{-7}\right)$, reached a suggestive level of association. We detected statistical heterogeneity in association with nevus count especially for IRF4, MTAP, PLA2G6, and DOCK8 (see Supplementary Tables 1 and 2) -that for IRF4 was expected-given our original studies of this gene showing crossover $\mathrm{G} \times$ age interaction. ${ }^{10}$ Meta-regression including age of the current study participants confirmed the age effect in the case of IRF4 (Supplementary Table 1).

Combining nevus and melanoma GWAS meta-analysesBayesian analysis. We then combined these nevus meta-analysis $P$ values with those from the melanoma meta-analysis ${ }^{10}$ (Table 1 , Fig. 2, Supplementary Figs 1,2). We used simple combination of $P$ values (weighted Stouffer method), as well as the GWAS-PW program, ${ }^{12}$ which combines GWAS data for two related traits to investigate the causes of genetic covariation between them (see Supplementary Methods). Specifically, it estimates Bayes factors and posterior probabilities of association (PPA) for four hypotheses: (a) a locus specifically affects melanoma only or (b) affects nevus count only; (c) a locus has pleiotropic effects on both traits; and (d) there are separate alleles at a locus independently determining each trait (colocation).

There were 30 regions containing SNPs that met our threshold for "interesting" (PPA > 0.5) for any of these hypotheses (Fig. 3, Supplementary Table 3). Twelve of these loci exhibited no evidence of association to nevus count, but were strongly associated with melanoma risk, one of the most extreme being $M C 1 R$. A total of 18 loci showed pleiotropic action with consistent directional and proportional effects of all SNPs on

Table 1 GWAS studies of nevus count contributing to the present meta-analysis

\begin{tabular}{|c|c|c|c|c|c|c|}
\hline Study & Nevus assessment & SNP chip & Imputation & Individuals (families) & $\begin{array}{l}\text { Age range } \\
\text { (mean) }\end{array}$ & Location (center) \\
\hline ALSPAC 39 & Self-count on limbs & $550 k$ & 1000Gv.3 & 3309 & $14-17(15.5)$ & UK (Bristol) \\
\hline Harvard 8 & $\begin{array}{l}\text { Self-count }>3 \mathrm{~mm} \text { on } \\
\text { limbs }\end{array}$ & $\begin{array}{l}\text { Affy+Illumina } \\
\text { various }\end{array}$ & 1000Gv.3 & 32,975 & $35-75(52)$ & US (Boston) \\
\hline Leeds 40 & $\begin{array}{l}\text { Whole-body count }>2 \\
\mathrm{~mm}\end{array}$ & OmniExpressExome & HRC v.1 & 397 & $21-80(57)$ & Yorkshire \\
\hline $\begin{array}{l}\text { QIMR BTNS } \\
\text { children }^{3}\end{array}$ & $\begin{array}{l}\text { Whole-body count }>0 \\
\mathrm{~mm}\end{array}$ & 610k, CoreExome & 1000Gv.3 & 3261 (1309) & $9-23(12.6)$ & $\begin{array}{l}\text { SE Queensland } \\
\text { (Brisbane) }\end{array}$ \\
\hline QIMR adult twins ${ }^{41}$ & Self-rating 4-point scale & $\begin{array}{l}317 k+370 k+610 k \\
+C E\end{array}$ & $1000 \mathrm{Gv} .3$ & $1848(1113)$ & $29--79(52.3)$ & Australia wide \\
\hline QIMR >50 twins 42 & $\begin{array}{l}\text { Self-count right arm }>4 \\
\mathrm{~mm}\end{array}$ & $370 k+610 k+C E$ & $1000 \mathrm{Gv} .3$ & $893(596)$ & $50-92(60.7)$ & Australia wide \\
\hline Raine $^{43}$ & Nurse-count right arm & $660 \mathrm{k}$ & $1000 \mathrm{Gv} .3$ & 808 & 22 & $\begin{array}{l}\text { Western Australia } \\
\text { (Perth) }\end{array}$ \\
\hline Twins UK 5 & $\begin{array}{l}\text { Whole-body count }>2 \\
\mathrm{~mm}\end{array}$ & $\begin{array}{l}317 \mathrm{k}+610 \mathrm{k}+1 \mathrm{M} \\
+1.2 \mathrm{M}\end{array}$ & 1000Gv.3 & 3312 (1839) & $18-80(47)$ & UK wide (London) \\
\hline Total nevus & & & & 52,506 & & \\
\hline Melanoma & & & & 12,874 cases; 23,203 & & \\
\hline GWASMA $^{10}$ & & & & controls & & \\
\hline Nevus+melanoma & & & & 88,583 (inc. controls) & & \\
\hline
\end{tabular}




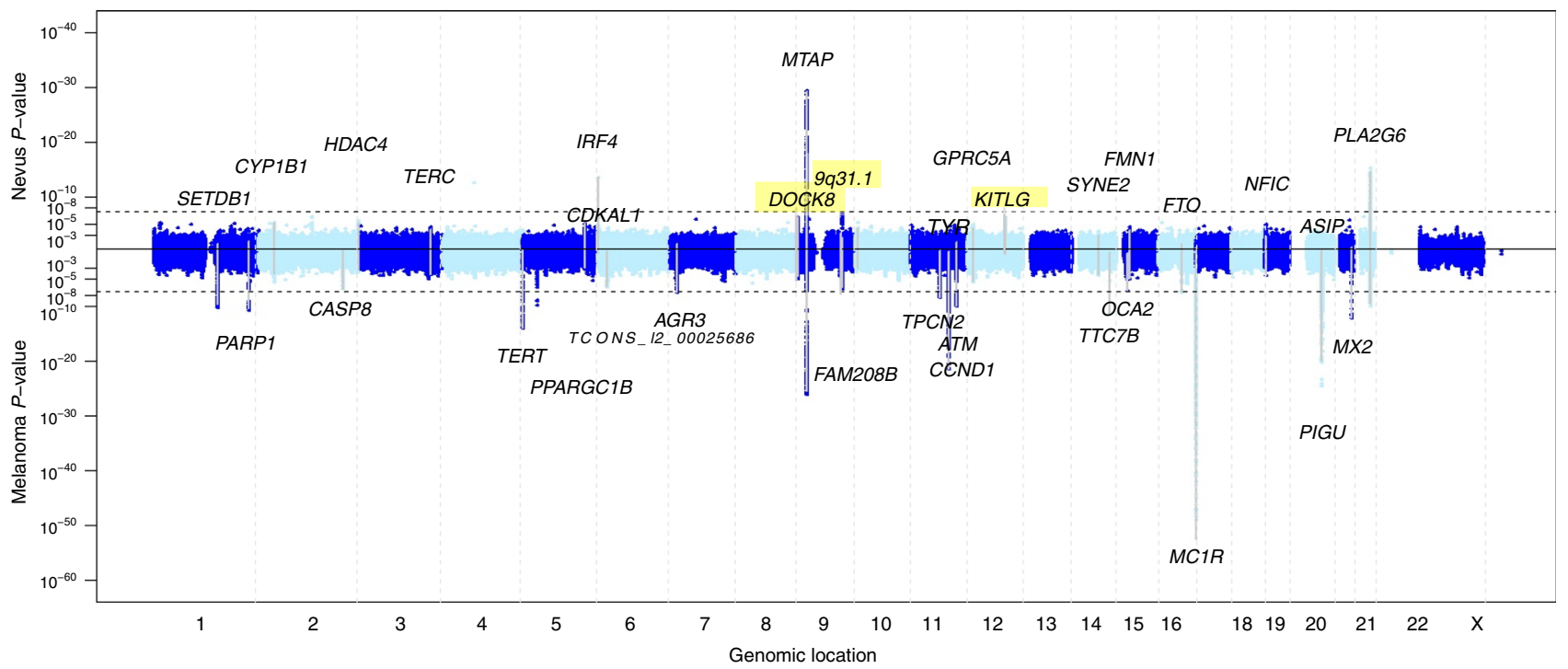

Fig. 1 Miami plot of nevus count and melanoma meta-analysis. $P$ values where either $P<10^{-5}$. The $-\log 10 P$ values for the nevus GWAS meta-analysis are above the central solid line and those for the melanoma GWAS meta-analysis are below that line. Novel nevus loci are highlighted

Table 2 SNPs associated with total nevus count and cutaneous melanoma (CM) in their respective meta-analyses

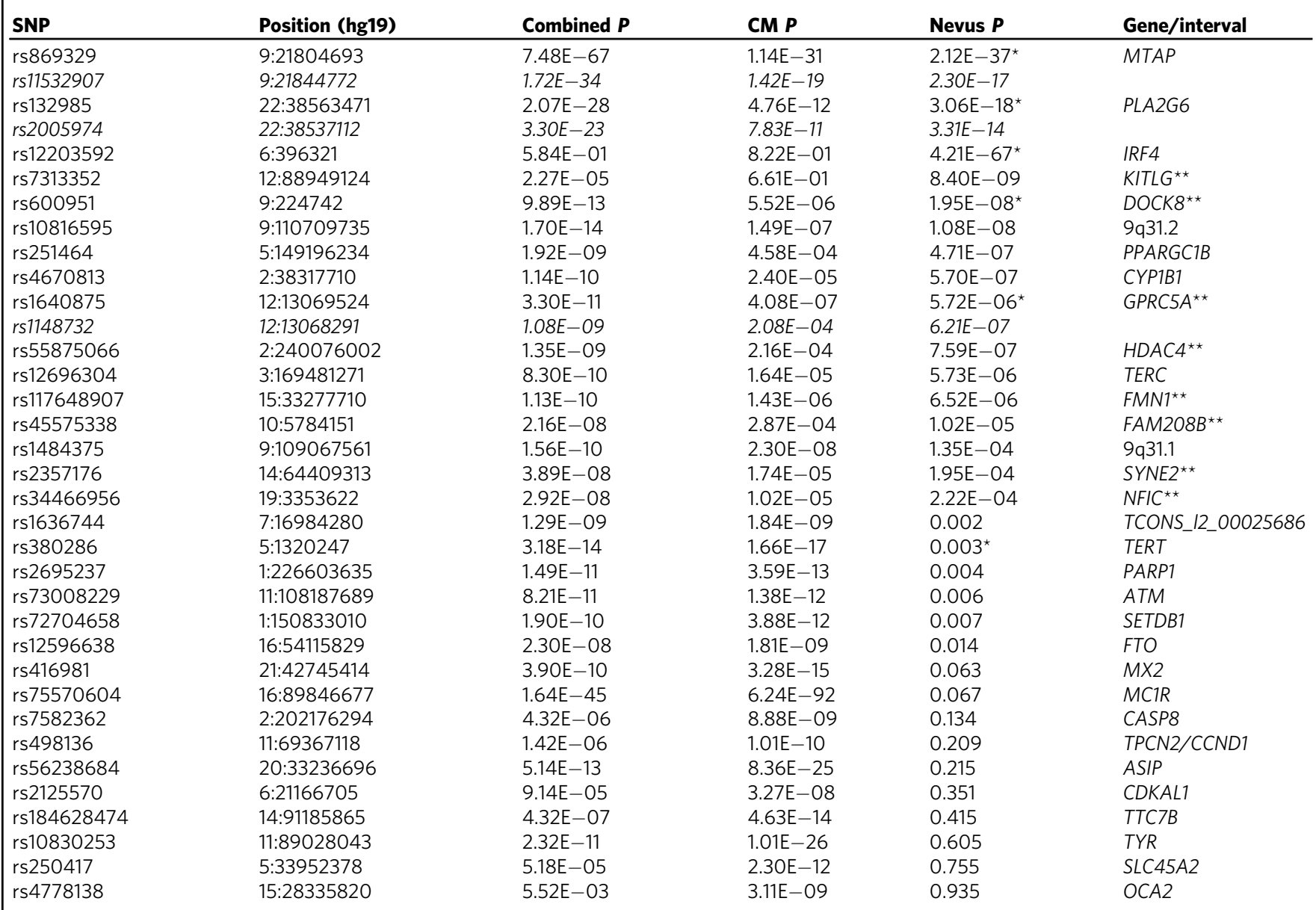

The weighted Stouffer method was used to combine the nevus and melanoma $P$ values (Combined $P$ ). The SNP with the smallest combined $P$ value under each peak is shown, but the table rows are ordered by strength of association to nevus count. In three cases where significant between-study heterogeneity is detected (unadjusted $P_{\text {hom }}<0.05$, denoted by ${ }^{*}$ ), the nevus $P$ value is from the random-effects model of $\mathrm{Han}$ and Eskin $^{38}$, and a result for a nearby SNP where $P_{\text {hom }}>0.05$ is included on the line beneath (italicized) to confirm genome-wide significance (in the case of IRF4 and DOCK8, there is no such nearby SNP).

*Unadjusted $P_{\text {hom }}<0.05$

${ }^{\star \star}$ Novel loci 


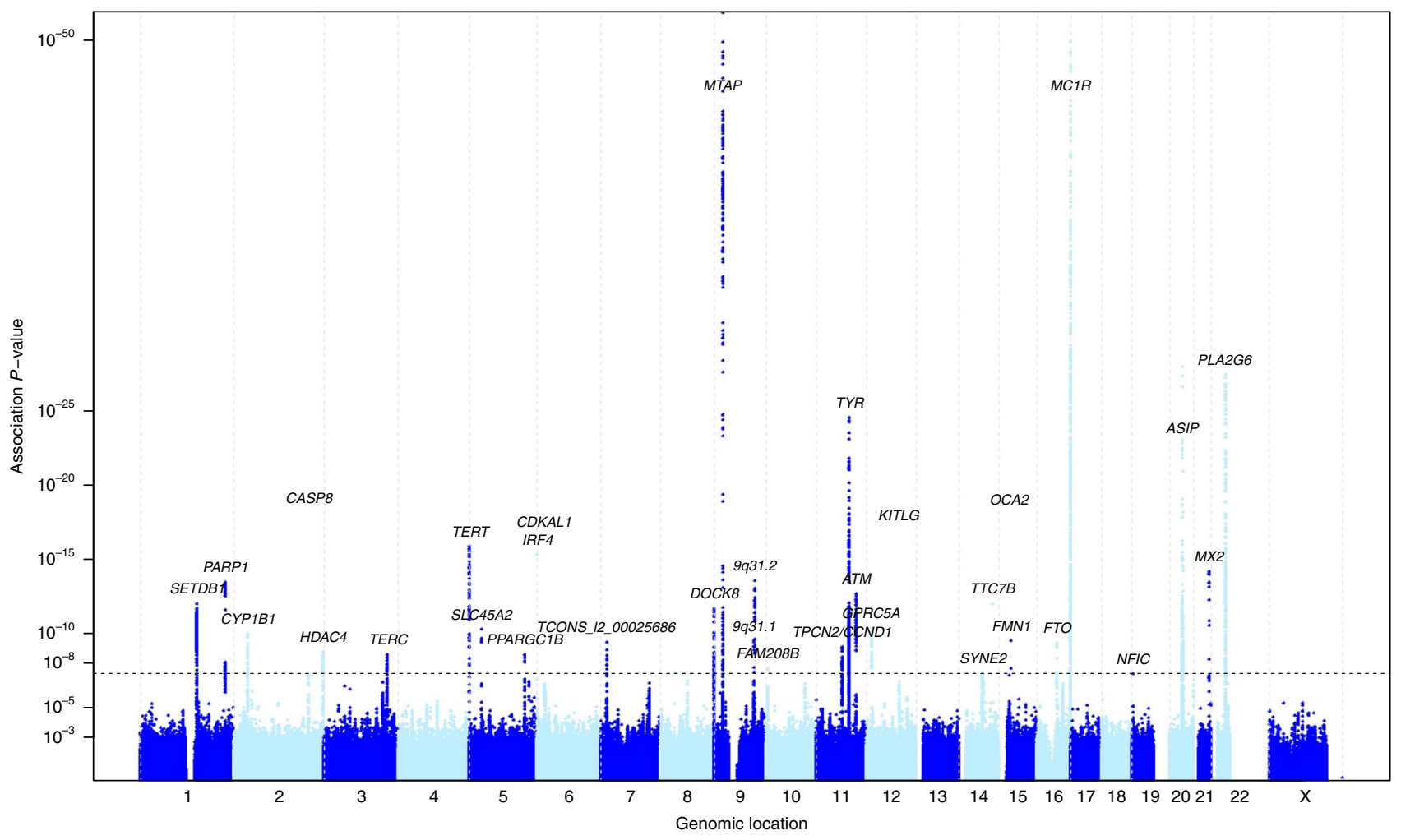

Fig. 2 Manhattan plot of $P$ values from meta-analysis combining nevus and melanoma results

nevi and melanoma risk, the strongest being MTAP, PLA2G6, and an intergenic region on $9 \mathrm{q} 31.1$ (Fig. 4 a shows a bivariate regional association around GPRC5A, all loci are shown in Supplementary Figs 5-19). There were no "pure nevus" regions using the binned GWAS-PW test (hypothesis b, PPAb > 0.2), with even the region of KITLG appearing as a pleiotropic region $(\mathrm{PPAb}=0.52$, PPAc $=0.11$ ), even though the pattern of bivariate association appears more consistent with a "nevus-only" locus (Fig. 4b). For another five regions, support was split between the pure melanoma and pleotropic models. In the case of IRF4, this is certainly driven by the marked between-study heterogeneity in melanoma association due to their different age distributions and latitudinal origins $^{13}$.

One interesting SNP (rs34466956), $2 \mathrm{kbp}$ upstream from NFIC on chromosome 19p13.3 (see Fig. 5), achieved a combined $P$ value of $3 \times 10^{-8}$ and a SNP-wise PPAc for pleiotropism of 0.9 , even though the binned GWAS-PW assigned the region a highest PPA of 0.28 .

Pleiotropy. The 18 pleiotropic loci each come from multiple pathways, indicating that nevogenesis is a more complicated process than previously anticipated. Pathways already implicated include those of MTAP (purine salvage pathway, possibly a rate limiting step to cell proliferation), PLA2G6 (phospholipase A2, implicated in apoptosis), and IRF4 (melanocyte pigmentation and proliferation). Newly implicated here in nevogenesis, TERC is a strong candidate given its involvement in telomere maintenance and prior suggestive evidence of association with melanoma/ nevi ${ }^{10,14,15}$, as well as several other cancers. ${ }^{16-18}$ PPARGC1B has previously been investigated as a skin color locus ${ }^{17}$ and there is functional evidence for its effects on melanocytes. ${ }^{18}$ GPRC5A (see Fig. 4a, Supplementary Fig. 15) has also been suggestively associated with melanoma ${ }^{10}$ and is a known oncogene in breast and lung cancer ${ }^{19,20}$. DOCK8 deficiency predisposes to virus-related malignancy and is deleted in some cancers, but not markedly in melanoma. ${ }^{21,22}$ DOCK8 regulates Cdc42 activation especially in immune effector cells-Cdc42 has been implicated in melanoma invasiveness $^{23}$ and variants in CDC42 have been previously associated with melanoma tumor thickness ${ }^{24}$ - though our best association $P$ value in the region of that latter gene is $3 \times 10^{-4}$.

The novel pleiotropic loci are: (a) the region around HDAC4 on chromosome 2; (b) chromosome 9q31 (two separate peaks); (c) near SYNE2 on chromosome 14; (d) in DOCK8 on chromosome 9p; and (e) near FMN1 on chromosome 15p (see Supplementary Results). For those loci that unequivocally lie within a gene, in each case that gene is expressed in melanocytes ${ }^{25}$ and these implicate several different pathways. The "master regulator" in melanocytogenesis ${ }^{26}$ is MITF (microphthalmia-associated transcription factor), and we confirmed that our top candidate genes in each of the 30 regions contain MITF binding sites. ${ }^{27}$ For example, three genes in the FMN1 region harbor MITF binding sites, viz. SCG5, RYR3, and FMN1 themselves (enrichment $P=$ 0.01). Furthermore, in several of these genes (MTAP, IRF4, PLA2G6, GPRC5A, and TERC), the most associated SNP lies within or close to the actual MITF binding sites, in some cases a rarer MITF-BRG1-SOX10-YY1 combined regulatory element $(\mathrm{MARE})^{27}$ (Supplementary Figs 20-40).

Gene based tests. The genes most strongly implicated in a genebased association analysis (PASCAL) are MTAP, PLA2G6, GPR5A, $A S B 13$ (adjacent to FAM208B), and KITLG $\left(P=2.3 \times 10^{-6}\right)$; see Supplementary Table 4). At a suggestive level, we note FAM208B, MGC16025 (both $\left.P=6 \times 10^{-6}\right)$, and HDAC4 $\left(1 \times 10^{-5}\right)$. Among genes at a significance level of $<10^{-4}$, we highlight $L M X 1 B(P=5 \times$ $10^{-5}$ ), where rs7854658 gave a nevus $P$ value of $3.3 \times 10^{-6}$.

Pathway analysis. Using different approaches (GWAS PRS, GWAS-PW, and REML using SNP sets; see Supplementary 

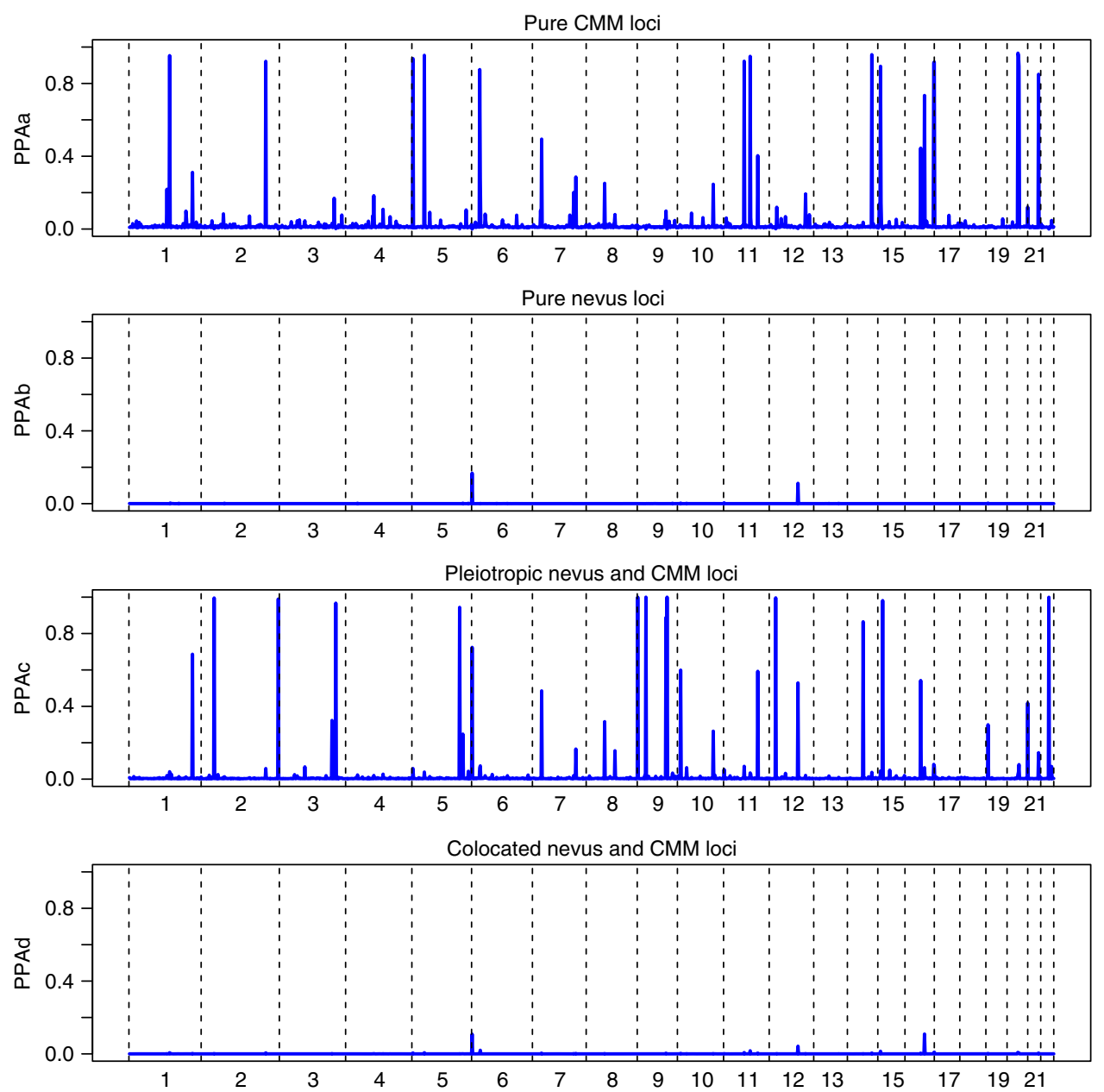

Fig. 3 Results of analyses using GWAS-PW, which assign posterior probabilities (PPA) to each of $\sim 1700$ genomic regions that is a a pure melanoma locus, b a pure nevus locus, $\mathbf{c}$ a pleiotropic nevus and melanoma loci, and $\mathbf{d}$ that the locus contains co-located but distinct variants for nevi and melanoma

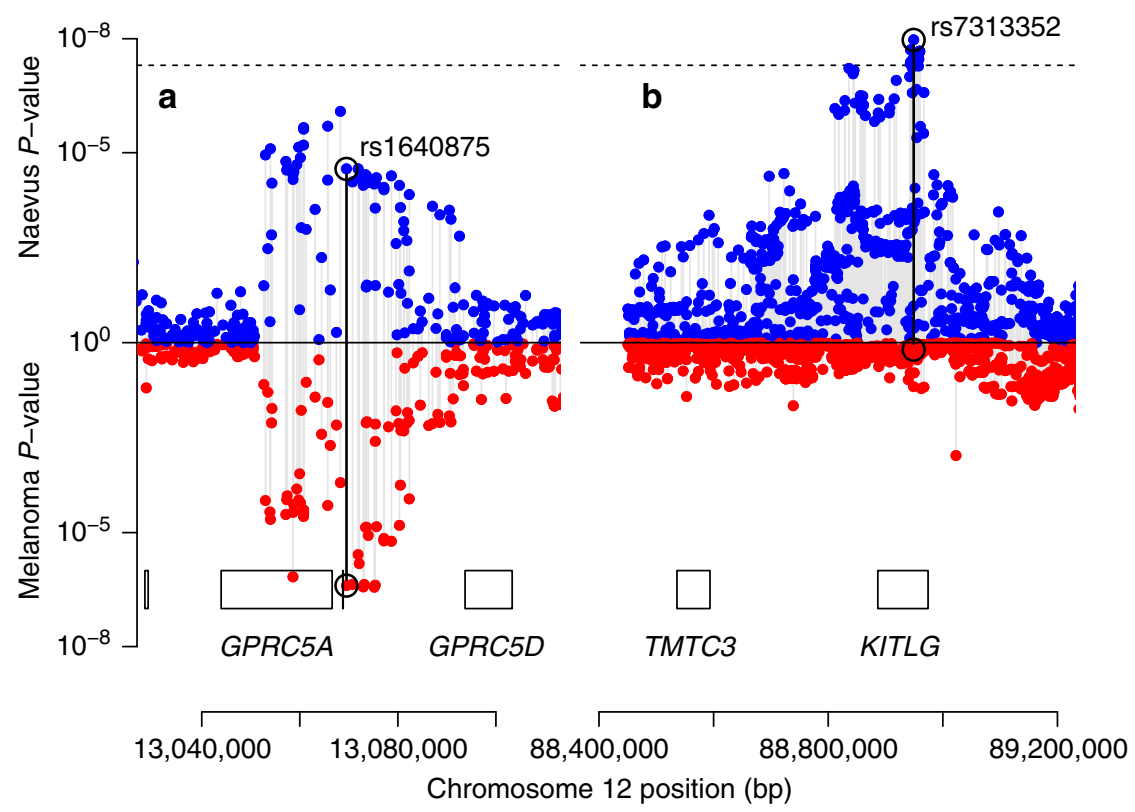

Fig. 4 Plot of nevus and melanoma association test $P$ values for a the region around rs1640875 in GPRC5A (chr12:12.9 Mbp) illustrating symmetrical influence on nevus count and melanoma risk; note that neither univariate peaks achieve significance alone but in combination they do (see Table 2, Fig. 2), and $\mathbf{b}$ the region around rs7313352 in KITLG (chr12:88.6 Mbp), a "pure" nevus locus with negligible direct effect on melanoma risk 


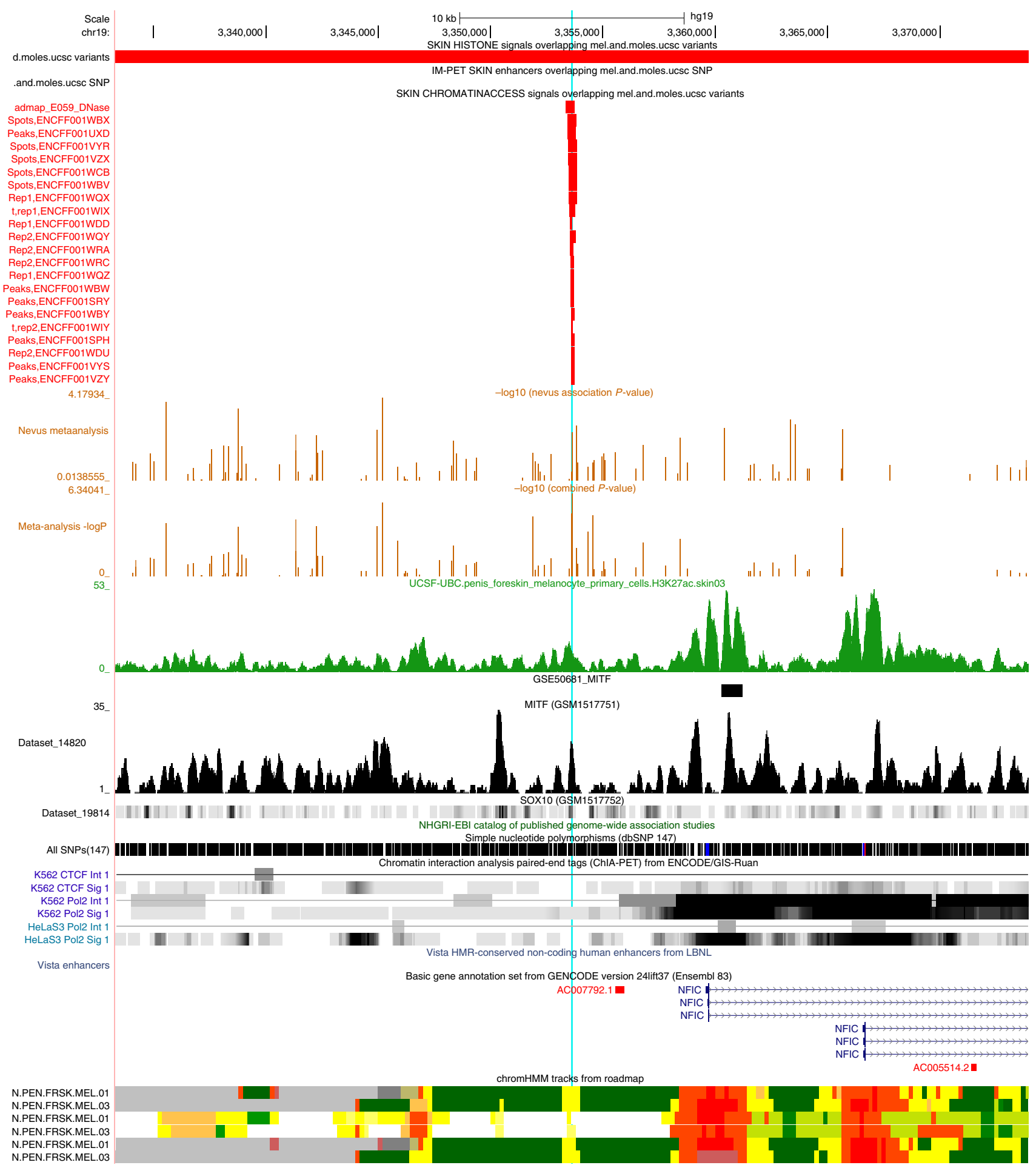

Fig. 5 UCSC Genome Browser view of region near NFIC (19p13.3). The pale blue line highlights location of rs34466956, which coincides with a narrow regulatory region as seen in in the 22 short red bars indicating open chromatin in melanocytes and skin. These align in the bottom 6 tracks with narrow yellow regions indicating results of hidden Markov models summarizing the evidence from multiple experiments for open chromatin in melanocytes. An MITF ChipSeq peak also overlies this same region (gray track, GSM1517751). NFIC is expressed in melanocytes, and a second larger MITF peak overlies intron 1 in two ChipSeq experiments viz. GSE50681_MITF, see short solid black bar, and also the tall sharp gray peak below it in GSM1517751.

See Supplementary Methods for details

Table 5), we tested candidate pathways ${ }^{28}$ for their overall contribution to variance in nevus number, the contribution of the telomere maintenance pathway was $0.8 \%$. A contribution of the immune regulation/checkpoint pathway was surprisingly absent, given our knowledge that immunosuppression increases nevus count quite promptly and the recent success of CTLA4 inhibitors in the treatment of melanoma. We did see a weak signal (Combined $P=1 \times 10^{-7}$ ) for rs870191, very close to SLE-associated SNPs just upstream from MIR146A, an important immune regulator. 
Genetic relationships with telomere length and pigmentation. In the GWAS-PW analysis combining melanoma and telomere length (TL) (see Supplementary Methods), there was considerable locus overlap, while by contrast only TERC was detectably shared between nevus count and TL (Supplementary Fig. 41). Note that SNPs in OBFC1 were only significantly associated with melanoma in the phase 2 analysis of Law et al. ${ }^{10}$ - which are not utilized in the GWAS-PW analysis-although they were suggestively associated $\left(P=10^{-5}\right)$ with nevus count. In the parallel analysis with pigmentation (indexed by dark hair color), only IRF4 overlapped with nevus count (Supplementary Fig. 42). Again, multiple pigmentation loci acted as risk factors for melanoma (with no overlap with TL). The fact that only TERC (and OBFC1) are associated with nevus count, while multiple loci are associated with melanoma, is not necessarily surprising. Telomere maintenance may predispose to melanoma directly as well as via nevus count, an extension of the "divergent pathway" hypothesis for melanoma ${ }^{29}$. However, the link with telomere length-associated SNPs may need a bigger sample size to look at associations further.

SNP heritability and genetic correlation. Mixed-model twin analyses with GCTA and LDAK (see Supplementary Methods) utilizing the Australian and British samples estimate the total heritability of nevus count to be $58 \%$ (and family environment $34 \%$ ), with contributions from every chromosome and one-sixth from chromosome 9 alone (see Supplementary Table 6). We found that $\sim 25 \%$ of the Australian and $\sim 15 \%$ of British genetic variance for nevus count could be explained by a panel of 1000 SNPs covering our 32 regions. We have also performed analyses examining the overall architecture of the relationship between nevus count and melanoma risk using bivariate LD score regression analysis and estimated $r_{\mathrm{g}}=0.69(S E=0.16)$ (see Supplementary Results). Alleles which increase nevus number proportionately increase the risk of melanoma (Supplementary Results, Supplementary Figs 43, 44) with KITLG, the interesting exception is that the nevus-associated variants did not predict melanoma risk (see Fig. 5b), rather, predisposing to other cancers (e.g., testicular germ cell).

\section{Discussion}

It has been long suggested that carrying out genetic analyses using multiple correlated phenotypes will increase power to detect trait loci in such a way as to justify the statistical complications. Since number of cutaneous nevus is strongly correlated with melanoma risk, and known nevus loci were associated with CM, it seemed likely that this would be a fruitful approach. We have highlighted eight novel loci, including the genes HDAC4, SYNE2, and most notably GPRC5A, where quite large samples of melanoma cases or nevus count were not sufficiently powerful to reach formal genome-wide significance in univariate analyses, but the combined evidence is conclusive.

Given that lighter skin color is also associated with both these phenotypes, we would expect a strong contribution from pigmentation pathway genes. Among those novel pleiotropic loci implicated in nevus count, $C Y P 1 B 1$ and $P P A R G C 1 B$ both appear in a recent skin pigmentation meta-analysis ${ }^{30}$ as harboring variants lightening skin color. The SNPs in the chromosome 7p21.1 region near $A H R$ and $A G R 3$ previously associated with $C M$ also appear to be associated with skin color in that study. In our analysis, the signal for nevus count from that interval (best $P=$ $3 \times 10^{-4}$ ) was half as strong as that for CM, and the GWAS-PW analysis support was equal for the hypotheses of a pure CM locus and a pleiotropic locus (region PPAa $=0.494$, PPAc $=0.485$ ). In passing, the peak SNPs lie within a long noncoding RNA gene
(TCONS_I2_00025688) that is expressed in melanocytes, so this is a potential candidate for both skin color and CM. In the case of KITLG, the variant most strongly associated with pigmentation (fair hair), rs12821256, modifies a distant enhancer, and was associated neither with melanoma or nevus count in our study (see Supplementary Results). We observe a similar pattern (association $P_{\text {nevus }}=0.4, P_{\mathrm{CM}}=0.8$ ) for the strongest associated variant for skin color from the skin color meta-analysis, rs $11104947 .{ }^{30}$

By contrast, HDAC4 and DOCK8 are in pathways that have not been implicated as important to nevogenesis or melanoma pathogenesis. HDAC4 is involved in transcriptional regulation in many tissues, while DOCK8 acts to regulate signal transduction, most notably in immune effector cells (see Supplementary Results). The association peak for HDAC4 is quite wide ( 80 $\mathrm{kbp}$ ), and overlaps with the multi-tissue GTEx eQTL peak for this gene. $^{31}$ The best overlapping SNP was rs115253975, with a combined nevus-CM $P$-value of $4 \times 10^{-9}$ and fibroblast HDAC4 eQTL $P$-value of $2 \times 10^{-5}$. The peak nevus-CMM DOCK8 SNP, rs600951, is a cis-eQTL in two (non-cutaneous) tissues, and the peak around it contains several eQTL SNPs detected in the GTEx skin samples. These eQTL SNPs would be potential causal candidates.

Both SYNE2 (encoding nesprin-2) and FMN1 (formin-1) are involved in nuclear envelope and cytoskeleton function, and through this in regulating as well as facilitating numerous biological pathways. Both, for example, are involved in directed cell migration. The nesprin and formin families have been implicated in efficient repair of double strand DNA breaks, so this might point to a mechanism for an association with nevi and CM (see Supplementary Results).

We did see heterogeneity between studies in strength of SNP association with nevus count or melanoma for four loci, most extremely for IRF4 (Supplementary Fig. 10). Meta-regression analysis suggested this is partly due to interactions with age in the case of IRF4 (Supplementary Table 1)-different nevus subtypes are known to predominate at different ages, with the dermoscopic globular type most common before age $20 .{ }^{32}$ We suspect sun exposure another important interacting covariate, given large differences in total nevus count by latitude. ${ }^{33,34}$

Epidemiologically, the etiology of melanoma has been divi$\operatorname{ded}^{35}$ into a chronic sun-exposure pathway and a nevus pathway, where intermittent sun exposure is sufficient to increase risk. At a genetic level, pigmentation genes such as $M C 1 R$ contribute only via the former pathway (though this can include effects on DNA repair ${ }^{36}$ ), others such as MTAP via the latter, while yet others such as IRF4 seem to act via both routes ${ }^{13}$. We interpret our results as consistent with the hypothesis that nevus number is the intermediate phenotype in a causative chain to melanoma originating in all these biologically heterogeneous nevus pathways. However, we acknowledge that there may also be some genes where there is a direct causal pathway to both phenotypes.

\section{Methods}

We carried out a meta-analysis of 11 sizeable GWAS of total nevus count in populations from Australia, Netherlands, Britain, and the United States, subsets of which have been reported on previously $y^{5,6,8}$, and then combined these results with those from a recently published meta-analysis of melanoma GWAS ${ }^{10}$ to increase power to detect pleiotropic genes. While nevus counts or density assessments are available for melanoma cases from a number of studies, in the meta-analysis of nevus count we included only samples of healthy individuals without melanoma, all of European ancestry (for more details, see Supplementary Methods).

Nevus phenotyping. The assessment of nevus counts varies considerably between the 11 studies in four respects (see Table 1): (a) nevus counts vs. density ratings; (b) whole body vs. only certain body parts; (c) all moles ( $>0 \mathrm{~mm}$ diameter) or only 
moles $>2 \mathrm{~mm}$, or $3 \mathrm{~mm}$, or $5 \mathrm{~mm}$; and (d) count by trained observer or self-count by study participant. These differences could contribute statistical heterogeneity into our analyses, so we have done considerable preliminary work to convince ourselves that all assessments are measuring the same biological dimension of "moliness" (see Supplementary Fig. 3). A pragmatic test of this is the relative contribution of each study to the detection of the known loci of large effect, which is evident from the forest plots (Supplementary Figs 5-19).

Statistical methods. Given this, we combined results from each study as regression coefficients and associated standard errors in standard fixed and random effects meta-analyses using the METAL ${ }^{37}$ and METASOFT ${ }^{38}$ programs. Manhattan and Q-Q plots for the nevus GWAS meta-analysis (GWASMA) are shown in Supplementary Fig. 45 and for each of the contributing studies in Supplementary Figs 46-55.

We combined the results from the nevus meta-analysis above with results from stage 1 of a recently published meta-analysis of $\mathrm{CM}^{10}$. Stage 1 of the $\mathrm{CM}$ study consisted of 11 GWAS data sets totaling 12,874 cases and 23,203 controls from Europe, Australia, and the United States; this stage included all six published CM GWAS and five unpublished ones. We do not utilize the results of stage 2 of that study, where a further $3116 \mathrm{CM}$ cases and 3206 controls from three additional data sets were genotyped for the most significantly associated SNP from each region, reaching $P<10^{-6}$ in stage 1 . As a result, certain melanoma association peaks are not genome-wide significant in their own right in the present bivariate analyses. Further details of these studies can be found in the Supplementary Note to Law et al. ${ }^{10}$. The combination of the nevus and melanoma results was performed using the Fisher method. A Manhattan plot for the combined nevus GWASMA plus melanoma GWASMA is shown in Supplementary Fig. 4. For more details of statistical methods, see Supplementary Methods.

\section{Data availability}

All relevant data are available from the authors upon application.

Received: 3 August 2017 Accepted: 13 September 2018.

Published online: 14 November 2018

\section{References}

1. Tucker, M. A. Melanoma epidemiology. Hematol. Oncol. Clin. North. Am. 23, 383-387 (2009).

2. Olsen, C. M., Carroll, H. J. \& Whiteman, D. C. Estimating the attributable fraction for cancer: a meta-analysis of nevi and melanoma. Cancer Prev. Res (Phila.) 3, 233-245 (2010).

3. Zhu, G. et al. A major quantitative-trait locus for mole density is linked to the familial melanoma gene CDKN2A: a maximum-likelihood combined linkage and association analysis in twins and their sibs. Am. J. Hum. Genet 65 483-492 (1999).

4. Wachsmuth, R. C. et al. Heritability and gene-environment interactions for melanocytic nevus density examined in a U.K. adolescent twin study. J. Invest Dermatol. 117, 348-352 (2001).

5. Falchi, M. et al. Genome-wide association study identifies variants at $9 \mathrm{p} 21$ and $22 \mathrm{q} 13$ associated with development of cutaneous nevi. Nat. Genet. 41, 915-919 (2009).

6. Duffy, D. L. et al. Multiple pigmentation gene polymorphisms account for a substantial proportion of risk of cutaneous malignant melanoma. J. Invest Dermatol. 130, 520-528 (2010).

7. Han, J. et al. A germline variant in the interferon regulatory factor 4 gene as a novel skin cancer risk locus. Cancer Res. 71, 1533-1539 (2011).

8. Nan, H. et al. Genome-wide association study identifies nidogen 1 (NID1) as a susceptibility locus to cutaneous nevi and melanoma risk. Hum. Mol. Genet 20, 2673-2679 (2011).

9. Duffy, D. L. et al. IRF4 variants have age-specific effects on nevus count and predispose to melanoma. Am. J. Hum. Genet 87, 6-16 (2010).

10. Law, M. H. et al. Genome-wide meta-analysis identifies five new susceptibility loci for cutaneous malignant melanoma. Nat. Genet. 47, 987-995 (2015).

11. Yang, J. et al. Genomic inflation factors under polygenic inheritance. Eur. J. Hum. Genet 19, 807-812 (2011).

12. Pickrell, J. K. et al. Detection and interpretation of shared genetic influences on 42 human traits. Nat. Genet 48, 709-717 (2016).

13. Gibbs, D. C. et al. Association of interferon regulatory factor-4 polymorphism rs12203592 with divergent melanoma pathways. J. Natl Cancer Inst. 108, djw004 (2016)

14. Bataille, V. et al. Nevus size and number are associated with telomere length and represent potential markers of a decreased senescence in vivo. Cancer Epidemiol. Biomark. Prev. 16, 1499-1502 (2007).

15. Iles, M. M. et al. The effect on melanoma risk of genes previously associated with telomere length. J. Natl Cancer Inst. 106, dju267 (2014).
16. Nan, H. et al. Genome-wide association study of tanning phenotype in a population of European ancestry. J. Invest Dermatol. 129, 2250-2257 (2009).

17. Shoag, J. et al. PGC-1 coactivators regulate MITF and the tanning response. Mol. Cell 49, 145-157 (2013)

18. Karami, S. et al. Telomere structure and maintenance gene variants and risk of five cancer types. Int J. Cancer 139, 2655-2670 (2016).

19. Sokolenko, A. P. et al. High prevalence of germline mutations in -mutant breast cancer patients. Int J. Cancer 134, 2352-2358 (2014).

20. Zhou, H. \& Rigoutsos, I. The emerging roles of GPRC5A in diseases. Oncoscience 1, 765-776 (2014).

21. Biggs, C. M. et al. DOCK8 deficiency: insights into pathophysiology, clinical features and management. Clin. Immunol. 181, 75-82 (2017).

22. Cancer Genome Atlas Network. et al. Genomic classification of cutaneous melanoma. Cell 61, 1681-1696 (2015).

23. Kedziora, K. M. et al. Rapid remodeling of invadosomes by Gi-coupled receptors: dissecting the role of Rho GTPases. J. Biol. Chem. 291, 4323-4333 (2016).

24. Vaysse, A. et al. A comprehensive genome-wide analysis of melanoma Breslow thickness identifies interaction between $C D C 42$ and SCIN genetic variants. Int J. Cancer 139, 2012-2020 (2016).

25. Reemann, P. et al. Melanocytes in the skin--comparative whole transcriptome analysis of main skin cell types. PLoS ONE 9, e115717 (2014).

26. Levy, C., Khaled, M. \& Fisher, D. E. MITF: master regulator of melanocyte development and melanoma oncogene. Trends Mol. Med 12, 406-414 (2006).

27. Laurette, P. et al. Transcription factor MITF and remodeller BRG1 define chromatin organisation at regulatory elements in melanoma cells. Elife 4, e06857 (2015).

28. Schoof, N., Iles, M. M., Bishop, D. T., Newton-Bishop, J. A. \& Barrett, J. H. Pathway-based analysis of a melanoma genome-wide association study: analysis of genes related to tumour-immunosuppression. PLoS ONE 6, e29451 (2011).

29. Whiteman, D. C. et al. Melanocytic nevi, solar keratoses, and divergent pathways to cutaneous melanoma. J. Natl. Cancer Inst. 95, 806-812 (2003).

30. Visconti, A. et al. Genome-wide association study in 176,678 Europeans reveals genetic loci for tanning response to sun exposure. Nat. Commun. 9 , 1684 (2018)

31. GTEx Consortium et al. Genetic effects on gene expression across human tissues. Nature 550, 204-213 (2017).

32. Zalaudek, I. et al. Age-related prevalence of dermoscopy patterns in acquired melanocytic naevi. Br. J. Dermatol. 154, 299-304 (2006).

33. Kelly, J. W. et al. Sunlight: a major factor associated with the development of melanocytic nevi in Australian schoolchildren. J. Am. Acad. Dermatol. 30, 40-48 (1994).

34. Bataille, V. et al. The association between naevi and melanoma in populations with different levels of sun exposure: a joint case-control study of melanoma in the UK and Australia. Br. J. Cancer 77, 505-510 (1998).

35. Shain, A. H. \& Bastian, B. C. From melanocytes to melanomas. Nat. Rev. Cancer 16, 345-358 (2016).

36. Wolf Horrell, E. M., Jarrett, S. G., Carter, K. M. \& D’Orazio, J. A. Divergence of cAMP signalling pathways mediating augmented nucleotide excision repair and pigment induction in melanocytes. Exp. Dermatol. 26, 577-584 (2017).

37. Willer, C. J., Li, Y. \& Abecasis, G. R. METAL: fast and efficient meta-analysis of genomewide association scans. Bioinforma. Bioinforma. 26, 2190-2191 (2010).

38. Han, B. \& Eskin, E. Random-effects model aimed at discovering associations in meta-analysis of genome-wide association studies. Am. J. Hum. Genet $\mathbf{8 8}$, 586-598 (2011).

39. Boyd, A. et al. Cohort Profile: the 'children of the 90s' - the index offspring of the Avon Longitudinal Study of Parents and Children. Int. J. Epidemiol. 42, 111-127 (2013)

40. Newton-Bishop, J. A. et al. Melanocytic Nevi, Nevus Genes, and Melanoma Risk in a Large Case-Control Study in the United Kingdom. Cancer Epidemiol. Biomark. Prev. 19, 2043-2054 (2010).

41. Werner, K. B. et al. The association between childhood maltreatment, psychopathology, and adult sexual victimization in men and women: results from three independent samples. Psychological Medicine 46, 563-573 (2016).

42. Mosing, M. A. et al. Genetic Influences on Life Span and Its Relationship to Personality: A 16-Year Follow-Up Study of a Sample of Aging Twins. Psychosomatic Medicine 74, 16-22 (2012).

43. Yazar, S. et al. Raine Eye Health Study: Design, Methodology and Baseline Prevalence of Ophthalmic Disease in a Birth-cohort Study of Young Adults. Ophthalmic Genetics 34, 199-208 (2013).

44. Hofman, A. et al. The Rotterdam Study: 2014 objectives and design update Eur. J. Epidemiol. 28, 889-926 (2013).

45. Mackey, D. A. et al. Twins Eye Study in Tasmania (TEST): Rationale and Methodology to Recruit and Examine Twins. Twin Research and Human Genetics 12, 441-454 (2009). 


\section{Author contributions}

S.C., J.H.B., N.K.H., S.M., A.H., M.K., D.H., J.A.N.B., T.D.S., D.M., G.D.S., T.E.N., D.T.B., V.B., M.F., J.H., and N.G.M. designed the study and obtained funding. G.Z., X.L., M.S., M.I., L.C.J., D.M.E., S.Y., J.B., M.L., P.K., A.V., J.C.T., F.L., J.A.M., and D.G. analyzed the data. M.J.W., E.N., and A.G. contributed to data collection and phenotype definitions. G. W.M., A.K.H., L.B., A.M.I., A.U., P.A.M., A.C.H., E.N., and M.B. contributed to genotyping. D.L.D. and N.G.M. wrote the first draft of the paper. All authors contributed to the final version of the paper.

\section{Additional information}

Supplementary Information accompanies this paper at https://doi.org/10.1038/s41467018-06649-5.

Competing interests: The authors declare no competing interests.

Reprints and permission information is available online at http://npg.nature.com/ reprintsandpermissions/
Publisher's note: Springer Nature remains neutral with regard to jurisdictional claims in published maps and institutional affiliations.

\begin{abstract}
Open Access This article is licensed under a Creative Commons

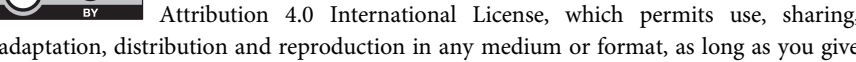
appropriate credit to the original author(s) and the source, provide a link to the Creative Commons license, and indicate if changes were made. The images or other third party material in this article are included in the article's Creative Commons license, unless indicated otherwise in a credit line to the material. If material is not included in the article's Creative Commons license and your intended use is not permitted by statutory regulation or exceeds the permitted use, you will need to obtain permission directly from the copyright holder. To view a copy of this license, visit http://creativecommons.org/ licenses/by/4.0/.
\end{abstract}

(C) The Author(s) 2018

\section{Melanoma GWAS Consortium}

Jeffrey E. Lee ${ }^{18}$, Myriam Brossard ${ }^{19}$, Eric K. Moses ${ }^{20}$, Fengju Song ${ }^{21}$, Rajiv Kumar ${ }^{22}$, Douglas F. Easton ${ }^{23}$, Paul D.P. Pharoah ${ }^{24}$, Anthony J. Swerdlow ${ }^{25}$, Katerina P. Kypreou ${ }^{26}$, Mark Harland ${ }^{27}$, Juliette Randerson-Moor ${ }^{27}$, Lars A. Akslen ${ }^{28}$, Per A. Andresen ${ }^{29}$, Marie-Françoise Avril ${ }^{30}$, Esther Azizi ${ }^{31}$, Giovanna Bianchi Scarrà ${ }^{32}$, Kevin M. Brown ${ }^{33}$, Tadeusz Dębniak ${ }^{34}$, David E. Elder ${ }^{35}$, Shenying Fang ${ }^{18}$, Eitan Friedman ${ }^{36}$, Pilar Galan ${ }^{37}$, Paola Ghiorzo ${ }^{32}$, Elizabeth M. Gillanders ${ }^{38}$, Alisa M. Goldstein ${ }^{33}$, Nelleke A. Gruis ${ }^{39}$, Johan Hansson ${ }^{40}$, Per Helsing ${ }^{41}$, Marko Hočevar ${ }^{42}$, Veronica Höiom ${ }^{40}$, Christian Ingvar ${ }^{43}$, Peter A. Kanetsky ${ }^{44}$, Wei V. Chen ${ }^{45}$, Maria Teresa Landi ${ }^{33}$, Julie Lang ${ }^{46}$, G. Mark Lathrop ${ }^{47}$, Jan Lubiński ${ }^{34}$, Rona M. Mackie ${ }^{48}$, Graham J. Mann ${ }^{49}$, Anders Molven ${ }^{50}$, Srdjan Novaković ${ }^{51}$, Håkan Olsson ${ }^{52}$, Susana Puig ${ }^{53}$, Joan Anton Puig-Butille ${ }^{53}$, Graham L. Radford-Smith ${ }^{54}$, Nienke van der Stoep ${ }^{55}$, Remco van Doorn ${ }^{39}$, David C. Whiteman ${ }^{56}$, Jamie E. Craig ${ }^{57}$, Dirk Schadendorf ${ }^{58}$, Lisa A. Simms ${ }^{55}$, Kathryn P. Burdon ${ }^{59}$, Dale R. Nyholt ${ }^{60}$, Karen A. Pooley ${ }^{23}$, Nicholas Orr ${ }^{61}$, Alexander J. Stratigos ${ }^{26}$, Anne E. Cust ${ }^{62,65}$, Sarah V. Ward ${ }^{20,66}$, Hans-Joachim Schulze ${ }^{63}$, Alison M. Dunning ${ }^{24}$, Florence Demenais ${ }^{19} \&$ Christopher I. Amos ${ }^{64}$

\footnotetext{
${ }^{18}$ Department of Surgical Oncology, The University of Texas MD Anderson Cancer Center, Houston, TX, USA. ${ }^{19}$ Institut National de la Santé et de la Recherche Médicale (INSERM), UMR-946, Genetic Variation and Human Diseases Unit, Paris, France. ${ }^{20}$ Centre for Genetic Origins of Health and Disease, Faculty of Medicine, Dentistry and Health Sciences, The University of Western Australia, Western Australia, Australia. ${ }^{21}$ Departments of Epidemiology and Biostatistics, Key Laboratory of Cancer Prevention and Therapy, National Clinical Research Center of Cancer, Tianjin Medical University Cancer Institute and Hospital, Tianjin 300060, P. R. China. ${ }^{22}$ Division of Molecular Genetic Epidemiology, German Cancer Research Center, Im Neuenheimer Feld 580, Heidelberg, Germany. ${ }^{23}$ Centre for Cancer Genetic Epidemiology, Department of Public Health and Primary Care, University of Cambridge, Cambridge, UK. ${ }^{24}$ Centre for Cancer Genetic Epidemiology, Department of Oncology, University of Cambridge, Cambridge, UK. ${ }^{25}$ Division of Genetics and Epidemiology, The Institute of Cancer Research, London, UK. ${ }^{26}$ Department of Dermatology, University of Athens School of Medicine, Andreas Sygros Hospital, Athens, Greece. ${ }^{27}$ Section of Epidemiology and Biostatistics, Leeds Institute of Cancer and Pathology, University of Leeds, Leeds, UK. ${ }^{28}$ Centre for Cancer Biomarkers CCBIO, Department of Clinical Medicine, University of Bergen, Bergen, Norway. ${ }^{29}$ Department of Pathology, Molecular Pathology, Oslo University Hospital, Rikshospitalet, Oslo, Norway. ${ }^{30}$ Assistance Publique-Hôpitaux de Paris, Hôpital Cochin, Service de Dermatologie, Université Paris Descartes, Paris, France. ${ }^{31}$ Department of Dermatology, Sheba Medical Center, Tel Hashomer, Sackler Faculty of Medicine, Tel Aviv, Israel. ${ }^{32}$ Department of Internal Medicine and Medical Specialities, University of Genoa, Genoa, Italy. ${ }^{33}$ Division of Cancer Epidemiology and Genetics, National Cancer Institute, National Institutes of Health, Bethesda, MD, USA.

${ }^{34}$ International Hereditary Cancer Center, Pomeranian Medical University, Czechs, Poland. ${ }^{35}$ Department of Pathology and Laboratory Medicine, Perelman School of Medicine at the University of Pennsylvania, Philadelphia, PA, USA. ${ }^{36}$ Oncogenetics Unit, Sheba Medical Center, Tel Hashomer, Sackler Faculty of Medicine, Tel Aviv University, Tel Aviv, Israel. ${ }^{37}$ Université Paris 13, Equipe de Recherche en Epidémiologie Nutritionnelle (EREN), Centre de Recherche en Epidémiologie et Statistiques, Institut National de la Santé et de la Recherche Médicale (INSERM U1153), Institut National de la Recherche Agronomique (INRA U1125), Conservatoire National des Arts et Métiers, Communauté d'Université Sorbonne Paris Cité, F-93017 Bobigny, France. ${ }^{38}$ Inherited Disease Research Branch, National Human Genome Research Institute, National Institutes of Health, Baltimore, MD, USA. ${ }^{39}$ Department of Dermatology, Leiden University Medical Centre, Leiden, The Netherlands. ${ }^{40}$ Department of Oncology-Pathology, Karolinska Institutet, Karolinska University Hospital, Stockholm, Sweden. ${ }^{41}$ Department of Dermatology, Oslo University Hospital, Rikshospitalet, Oslo, Norway.
} 
${ }^{42}$ Department of Surgical Oncology, Institute of Oncology Ljubljana, Ljubljana, Slovenia. ${ }^{43}$ Department of Surgery, Clinical Sciences, Lund University, Lund, Sweden. ${ }^{44}$ Department of Cancer Epidemiology, H. Lee Moffitt Cancer Center and Research Institute, Tampa, FL, USA. ${ }^{45}$ Department of Genetics, The University of Texas MD Anderson Cancer Center, Houston, TX, USA. ${ }^{46}$ Department of Medical Genetics, University of Glasgow, Glasgow, UK. ${ }^{47}$ McGill University and Genome Quebec Innovation Centre, Montreal, Canada. ${ }^{48}$ Department of Public Health, University of Glasgow, Glasgow, UK. ${ }^{49}$ Centre for Cancer Research, University of Sydney at Westmead, Millennium Institute for Medical Research and Melanoma Institute Australia, Sydney, Australia. ${ }^{50}$ Department of Pathology, Haukeland University Hospital, Bergen, Norway. ${ }^{51}$ Department of Molecular Diagnostics, Institute of Oncology Ljubljana, Ljubljana, Slovenia. ${ }^{52}$ Department of Oncology/Pathology, Clinical Sciences, Lund University, Lund, Sweden. ${ }^{53}$ Melanoma Unit, Dermatology Department \& Biochemistry and Molecular Genetics Departments, Hospital Clinic, Institut de Investigacó Biomèdica August Pi Suñe, Universitat de Barcelona, Barcelona, Spain. ${ }^{54}$ Inflammatory Bowel Diseases, QIMR Berghofer Medical Research Institute, Brisbane, Australia. ${ }^{55}$ Department of Clinical Genetics, Leiden University Medical Center, Leiden, The Netherlands. ${ }^{56}$ Cancer Control Group, QIMR Berghofer Medical Research Institute, Brisbane, Australia. ${ }^{57}$ Department of Ophthalmology, Flinders University, Adelaide, Australia. ${ }^{58}$ Department of Dermatology, University Hospital Essen, Essen, Germany. ${ }^{59}$ Menzies Institute for Medical Research, University of Tasmania, Hobart, TAS, Australia. ${ }^{60}$ Institute of Health and Biomedical Innovation, Queensland University of Technology, Brisbane, QLD, Australia. ${ }^{61}$ Breakthrough Breast Cancer Research Centre, The Institute of Cancer Research, London, UK. ${ }^{62}$ Cancer Epidemiology and Services Research, Sydney School of Public Health, The University of Sydney, Sydney, Australia. ${ }^{63}$ Department of Dermatology, Fachklinik Hornheide, Institute for Tumors of the Skin at the University of Münster, Münster, Germany. ${ }^{64}$ Department of Community and Family Medicine, Geisel School of Medicine, Dartmouth College, Hanover, NH, USA. ${ }^{65}$ Sydney School of Public Health and the Melanoma Institute Australia, University of Sydney, Sydney, Australia. ${ }^{66}$ Department of Epidemiology and Biostatistics, Memorial Sloan Kettering Cancer Center, New York, USA 